\title{
Long-Term Study about the Incidence of Epilepsy in Male Service Personnel from India: A Retrospective, Cohort Study
}

\author{
Pawan Dhull $^{1} \quad$ S. K. Patnaik ${ }^{2}$ Manoj Somasekharan ${ }^{1} \quad$ K. V. S. Hari Kumar \\ ${ }^{1}$ Department of Neurology, Army Hospital (R\&R), Delhi, India \\ 2Department of Pediatrics, Army Hospital (R\&R), Delhi, India \\ ${ }^{3}$ Department of Endocrinology, Army Hospital (R\&R), Delhi, India \\ Address for correspondence K.V. S. Hari Kumar, MD, DNB, \\ Department of Endocrinology, Army Hospital (R\&R), Delhi, India \\ (e-mail: hariendo@rediffmail.com).
}

J Neurosci Rural Pract 2019;10:588-591

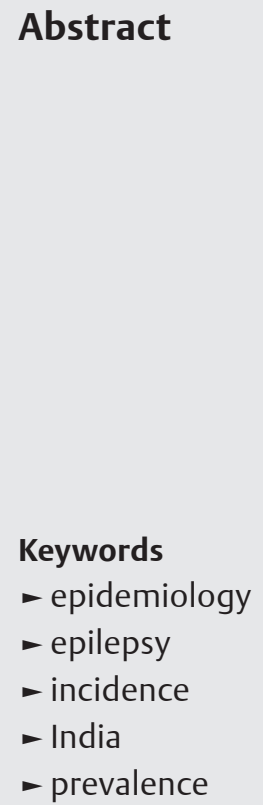

Background The data on the epidemiology of epilepsy are limited for developing countries including India. We estimated the incidence of epilepsy in a cohort of service personnel from India followed for over two decades.

Materials and Methods The data for this epidemiological study were derived from the electronic medical records (EMRs) of the male service personnel. The participants (age < 18 years) were enrolled into active service between 1990 and 2015 in good health. The data pertaining to the diagnosis of epilepsy were derived from the EMR using the prevalent International Classification of Diseases codes. We calculated the incidence rate as per person-years (py) using appropriate statistical methods.

Results Our data included 51,217 participants (median age: 33 years, range: 17-54) with a mean follow-up of 12.5 years, giving a cumulative follow-up duration of 613,925 py. A total of 291 patients developed epilepsy during the study, giving an incidence rate of 0.47 per 1000 py (95\% confidence interval: $0.42-0.53$ ). Undifferentiated spondyloarthropathy, central nervous system disorders, and alcohol dependence syndrome were the common comorbid ailments in patients with epilepsy.

Conclusion Our cohort had a comparable incidence rate of epilepsy with other studies from India and abroad.

\section{Introduction}

Epilepsy is one of the common disorders encountered in the practice of neurology. Epilepsy is estimated to be present in $\sim 1 \%$ of the Indian population, giving an estimated number of patients in excess of 13 million patients. ${ }^{1}$ The most common etiologies of epilepsy include the central nervous system (CNS) infections, tumors, neurotrauma, and genetic syndromes. ${ }^{2} \mathrm{~A}$ few types of epilepsy have been described in particular geographic regions, namely "hot water epilepsy" from South India. ${ }^{3}$ The pattern and presentation of the epilepsy are similar between the developed and developing countries. However, certain regional disparities exist between the urban and rural regions about the epidemiological determinants of the epilepsy. ${ }^{4}$ The epilepsy is often underdiagnosed and treated inadequately in the rural parts of India.
The epidemiological studies from India about the epilepsy are scarce and limited for many reasons. ${ }^{5}$ First, the diagnosis is often confused between the syncope and pseudoseizures. Second, the patients are completely normal in the interictal period leading to misdiagnosis. Third, the associated stigma leads to underreporting of the epilepsy, especially from the rural areas. ${ }^{6}$ Finally, the diagnosis and classification of epilepsy and seizures are not uniform among physicians leading to poor data compilation. A comprehensive epidemiological data are essential to identify the disease burden in the community and allocate the resources. A limited number of studies exist from India that have described the incidence rate of epilepsy in the community. ${ }^{7-9}$ However, these studies are limited by their sample size, distribution, and the duration of observation. Hence, we conducted this study to assess 
the incidence rate (IR) of epilepsy in a cohort of service personnel followed for a long duration.

\section{Materials and Methods}

\section{Study Population}

We conducted this retrospective study using the electronic medical records' (EMRs) data pertaining to the personnel of our organization. The detailed methodology about the study population and procedure was described in an earlier study giving the incidence of parathyroid disorders. ${ }^{10}$ Our organization consists of $\geq 55,000$ health-care personnel (HCP) working at various primary-, secondary-, and tertiary-level health-care centers. The participants of this study ( $n=51,217)$ were enrolled in the active military service between January 1, 1990, and December 31, 2015. They were recruited before the age of 18 years in good physical and mental health. Each HCP is assigned a unique number that can be used for the retrieval of the data from the EMR database. The study population is educated below graduate level and is derived from all the regions and states of our country. Our organization does not enlist females in the personnel below officer rank, thereby limiting our data to males only.

\section{Study Procedure}

The EMR provided the data on occupational and demographic particulars that include age, education, marital status, and medical conditions. The sickness and hospitalization records of all the HCP are captured in the EMR database. The data were entered into the EMR database at the respective health centers dealing with the patients. The HCP trained in the database management entered the details after perusing the clinical record of the patient given by the treating specialist. The data were derived from the central database and we did not have data monitoring teams at the peripheral centers. All disorders that require long-term treatment are reviewed periodically under observation. The prevailing International Classification of Diseases (ICD) codes were used to enter the disorders in the EMR data. We used the code numbers 345 and G40 of ICD 9 and 10, respectively, for the diagnosis of epilepsy. In patients with epilepsy, we searched for any comorbid ailments using the patient-specific identification number. We have not perused the individual medical records, and the diagnosis of epilepsy was based on the EMR only. We have included the first episode of epilepsy only for the purpose of this study, as the same is useful in the calculation of incidence rate.

\section{Statistical Analysis}

The incidence rate was calculated as the number of new cases diagnosed per year divided by the total number of person-years (py) follow-up. The index year was noted based on the first entry of the medical condition in the record of the individual. Other demographic data were presented using mean, standard deviation, and percentage.

\section{Results}

The study participants consist of 51,217 males (median age: 33 years, range: 17-54) followed up for a mean duration of 12.5 years. Our data gave a total 613,925 py duration of the follow-up period. Epilepsy was diagnosed in 291 patients, giving an incidence rate of 0.47 per 1000 py ( $95 \%$ confidence interval between 0.42 and 0.53 ). The mean age of the patients at the time of diagnosis of epilepsy was $27.9 \pm 5.5$ years. The mean duration of follow-up after the diagnosis was $8.3 \pm$ 6.7 years. A total of 198 patients with epilepsy had comorbid ailments as shown in $\boldsymbol{- F i g}$. 1. The top four comorbid ailments observed in these patients include undifferentiated spondyloarthropathy, CNS disorders, alcohol dependence syndrome (ADS), and fractures as shown in - Table 1. Our data do not give any information about the deceased individuals and the contribution of the epilepsy to the mortality.

\section{Discussion}

Our data showed an incidence rate of 0.47 per 1000 py of epilepsy in the service population observed for over two decades. Previous studies from India have shown that the incidence rate varied between 0.2 and 0.6 per 1000 population. ${ }^{11}$ Two studies from the rural parts of India have given an

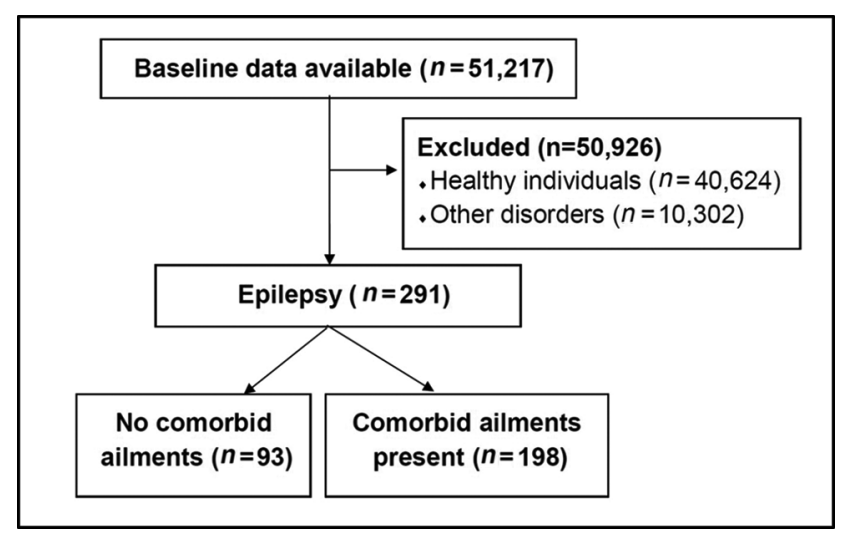

Fig. 1 Flow diagram of the study.

Table 1 Comorbid ailments in epilepsy patients

\begin{tabular}{|l|l|}
\hline Disorder & Total $=198, \boldsymbol{n}(\%)^{\mathrm{a}}$ \\
\hline $\begin{array}{l}\text { Undifferentiated } \\
\text { spondyloarthropathy }\end{array}$ & $134(67.7)$ \\
\hline CNS disorders & $28(14.1)$ \\
\hline Alcohol dependence syndrome & $27(13.6)$ \\
\hline Fractures (any site) & $27(13.6)$ \\
\hline Psychiatric disorders & $19(9.6)$ \\
\hline Hypertension & $11(5.6)$ \\
\hline Diabetes mellitus & $6(3)$ \\
\hline Others & $63(31.8)$ \\
\hline
\end{tabular}

Abbreviation: CNS, central nervous system.

aThe sum total is $>198$, as few patients have multiple disorders. 
incidence rates of 0.47 and 0.49 per 1000 population. ${ }^{7,8}$ Our data pertain to males only, whereas the other studies involve patients of both genders, precluding a meaningful comparison with our data. Sawhney et al have reported a higher incidence rate (6 per 1000) in the urban migrant population. ${ }^{12}$ The incidence rate derived from our data is in the expected lines with other studies and also eliminates the bias associated with the previously reported data from the urban area. Another study from the urban area has shown an incidence rate similar to that of our study, though with a smaller sample. ${ }^{9}$ In our data, the mean age of presentation of epilepsy is in the third decade. Previous reports suggest a bimodal distribution of age with the peaks in the first and sixth decade. ${ }^{13}$ However, our study sample includes patients beyond 18 years of age only, thereby limiting the comparison with other studies. Recent reviews have suggested an increasing prevalence in the second to fourth decade due to the demographic transition of the population. ${ }^{14}$ Previous reports suggest a slightly higher prevalence rates in the male gender. ${ }^{15}$ We are unable to comment on gender differences as our data do not include any females.

Another important finding of our study is the associated comorbid ailments in the patients with epilepsy. Undifferentiated spondyloarthropathy is the most common ailment seen in $68 \%$ of patients with epilepsy. This could have been contributed by the associated occult vitamin D deficiency (VDD) as explained below: First, VDD is very common in India and they present with osteomalacia and myalgia. ${ }^{16}$ Second, the use of antiepileptic drugs (AEDs) such as valproate and phenytoin results in the VDD, aggravating the symptoms. ${ }^{17}$ Third, vitamin $\mathrm{D}$ is not routinely supplemented in our patients using an AED. Finally, the food products and milk are not fortified with vitamin D in India, leading to significant VDD in the general population. ADS is the third common disorder seen in our patients with epilepsy. Our study population, being derived from the military service, has a higher prevalence of alcohol consumption in comparison to the general population. ${ }^{18}$ However, the EMR includes the data about the patients who fulfill the diagnostic criteria for ADS only and excludes the patients with binge drinking and other forms of alcohol consumption.

Neuroinfections, neurotrauma, and metabolic conditions are the common etiologies of the epilepsy from India. ${ }^{19} \mathrm{How}-$ ever, our data suggest idiopathic (32\%) as the most common disorder followed by neuroinfections (14\%), alcohol (14\%), and psychiatric disorders (10\%). The discrepancy in our database could be explained by the relatively young population, good socioeconomic status, and the possibility of exclusion of certain infections separately in the database. Neurocysticercosis (NCC) is a common cause of epilepsy, and in a typical case, the treating physician could have labeled the individual as only a case of epilepsy without giving a separate diagnosis of NCC. ${ }^{20}$ We have not perused the individual medical records of the patients, to identify the exact etiology and the type of the epilepsy. The strengths of our study include longer duration of observation and robust follow-up of a sizeable number of patients. The absence of data pertaining to the female sex and possible clerical errors in data entry leading to misdiagnosis of ICD codes are certain limitations of our study. Another limitation is the lack of information about the HCPs who are treated outside our organization resulting in low IR of the epilepsy. We have not observed the participants before their entry into the military service to rule out a preexisting epilepsy, thereby resulting in another limitation of our study.

\section{Conclusion}

Our report showed the incidence rates of epilepsy from a large service population of India. Our data are comparable with that of the other published reports on the epilepsy and help in the reliable estimation of the disease burden in the community.

\section{Funding}

None.

\section{Conflict of Interest}

None declared.

\section{References}

1 Santhosh NS, Sinha S, Satishchandra P. Epilepsy: Indian perspective. Ann Indian Acad Neurol 2014;17(Suppl 1):S3-S11

2 Kalita J, Nair PP, Misra UK. A clinical, radiological and outcome study of status epilepticus from India. J Neurol 2010;257(2):224-229

3 Satishchandra P, Shivaramakrishana A, Kaliaperumal VG, Schoenberg BS. Hot-water epilepsy: a variant of reflex epilepsy in southern India. Epilepsia 1988;29(1):52-56

4 Gourie-Devi M, Gururaj G, Satishchandra P. Subbakrishna DK. Prevalence of neurological disorders in Bangalore, India: a community-based study with a comparison between urban and rural areas. Neuroepidemiology 2004;23(6):261-268

5 Gourie-Devi M, Gururaj G, Satishchandra P, Subbakrishna DK. Neuro-epidemiological pilot survey of an urban population in a developing country. A study in Bangalore, south India. Neuroepidemiology 1996;15(6):313-320

6 Asawavichienjinda T, Sitthi-Amorn C, Tanyanont W. Compliance with treatment of adult epileptics in a rural district of Thailand. J Med Assoc Thai 2003;86(1):46-51

7 Mani KS, Rangan G, Srinivas HV, Kalyanasundaram S, Narendran S, Reddy AK. The Yelandur study: a community-based approach to epilepsy in rural South India-epidemiological aspects. Seizure 1998;7(4):281-288

8 Saha SP, Bhattachrya S, Roy BK, et al. A prospective incidence study of epilepsy in a rural community of West-Bengal, India. Neurol Asia 2008;13:41-48

9 Banerjee TK, Ray BK, Das SK, et al. A longitudinal study of epilepsy in Kolkata, India. Epilepsia 2010;51(12):2384-2391

10 Hari Kumar KVS, Patnaik SK. Incidence of parathyroid disorders in Indian adult male population: a 25-year follow-up study. Clin Endocrinol (Oxf) 2017;87(5):605-608

11 Amudhan S, Gururaj G, Satishchandra P. Epilepsy in India I: epidemiology and public health. Ann Indian Acad Neurol 2015;18(3):263-277

12 Sawhney IM, Singh A, Kaur P, Suri G, Chopra JS. A case control study and one year follow-up of registered epilepsy cases in a resettlement colony of North India, a developing tropical country. J Neurol Sci 1999;165(1):31-35

13 Hauser WA, Annegers JF, Kurland LT. Incidence of epilepsy and unprovoked seizures in Rochester, Minnesota: 1935-1984. Epilepsia 1993;34(3):453-468 
14 Gourie-Devi M. Epidemiology of neurological disorders in India: review of background, prevalence and incidence of epilepsy, stroke, Parkinson's disease and tremors. Neurol India 2014;62(6):588-598

15 Radhakrishnan K, Pandian JD, Santhoshkumar T, et al. Prevalence, knowledge, attitude, and practice of epilepsy in Kerala, South India. Epilepsia 2000;41(8):1027-1035

16 G R, Gupta A. Vitamin D deficiency in India: prevalence, causalities and interventions. Nutrients 2014;6(2):729-775

17 Menon B, Harinarayan CV. The effect of anti epileptic drug therapy on serum 25-hydroxyvitamin D and parameters of calcium and bone metabolism-a longitudinal study. Seizure 2010;19(3):153-158

18 Bray RM, Marsden ME, Peterson MR. Standardized comparisons of the use of alcohol, drugs, and cigarettes among military personnel and civilians. Am J Public Health 1991;81(7):865-869

19 Murthy JM, Yangala R, Srinivas M. The syndromic classification of the International League Against Epilepsy: a hospital-based study from South India. Epilepsia 1998;39(1):48-54

20 Carpio A, Romo ML. The relationship between neurocysticercosis and epilepsy: an endless debate. Arq Neuropsiquiatr 2014;72(5):383-390 